\title{
PARALLELISM IN THE TENSOR ANALYSIS OF PARTIAL DIFFERENTIAL EQUATIONS
}

\author{
D. D. KOSAMBI
}

In the extensions of classical differential geometry associated with ordinary differential equations the connection coefficients may be chosen arbitrarily, subject to certain laws of transformation under change of coordinates. But the calculations of differential invariants become decidedly simpler if the choice is made in a particular way, so as to reduce the number of "invariants of the connection" to a minimum, leaving only the intrinsic invariants of the system. For partial differential equations, no such intrinsic choice had hitherto been found, though something of the sort is badly needed inasmuch as the mass of calculations is far more complicated than for any system of ordinary differential equations.

Given the system of partial differential equations

$$
\begin{gathered}
\frac{\partial^{\alpha+1} x^{i}}{\partial u^{\alpha_{1}} \cdots \partial u^{\alpha_{q}+1}}+H_{\alpha_{1} \cdots \alpha_{q+1}}^{i}\left(u, x, p_{\alpha_{1}}^{i} \cdots p_{\alpha_{1}}^{i}, \cdots, \alpha_{q}\right)=0 ; \\
p_{\alpha}^{i}=\frac{\partial x^{i}}{\partial u^{\alpha}}, \cdots, p_{\alpha_{1} \cdots \alpha_{q}}^{i}=\frac{\partial p_{\alpha_{1}}^{i} \cdots \alpha_{q-1}}{\partial u^{\alpha_{q}}}, \\
i, j, k, \cdots=1,2, \cdots, n ; \alpha, \beta, \gamma, \cdots=1,2, \cdots, m,
\end{gathered}
$$

the complete set of tensor operators and tensor differential invariants associated with the "path-space" of this system may be built up as follows. We assume that (a) the equations (1) have a manifold of solutions; (b) they are tensorial under nonsingular point-transformations in both the $x$ and the $u$ coordinates; (c) the equations both of the $x$-variation and the $u$-variation of (1) are tensor-invariant and (d) there exists a tensorial differentiation operator

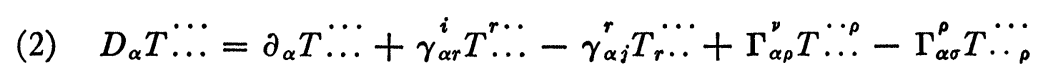

(one summation for every index of the tensor $T_{\ldots} .$. ), where

$$
\partial_{\alpha}=\frac{\partial}{\partial u^{\alpha}}+p_{\alpha}^{i} \frac{\partial}{\partial x^{i}}+p_{\alpha \beta}^{i} \frac{\partial}{\partial p_{\beta}^{i}}+\cdots-H_{\alpha \beta_{1} \cdots \beta_{q}}^{i} \frac{\partial}{\partial p_{\beta_{1}}^{i} \cdots \beta_{q}} .
$$

For the tensor invariance of this operator, it is necessary and sufficient that the connection coefficients $\gamma_{\alpha j}^{\beta}, \Gamma_{\beta \nu}^{\alpha}$ obey the transformation laws:

Received by the editors April 22, 1944, and, in revised form, December 13, 1944. 


$$
\left\{\begin{aligned}
\gamma_{\sigma j}^{s} \frac{\partial \bar{x}^{i}}{\partial x^{s}} \frac{\partial u^{\sigma}}{\partial \bar{u}^{\alpha}} & =\bar{\gamma}_{\alpha s}^{i} \frac{\partial \bar{x}^{s}}{\partial x^{j}}+\dot{p}_{\sigma}^{s} \frac{\partial^{2} \bar{x}^{i}}{\partial x^{j} \partial x^{s}} \frac{\partial u^{\sigma}}{\partial \bar{u}^{\alpha}} \\
\bar{\Gamma}_{\alpha \beta}^{\sigma} \frac{\partial u^{\nu}}{\partial \bar{u}^{\sigma}} & =\Gamma_{\sigma \tau}^{\nu} \frac{\partial u^{\sigma}}{\partial \bar{u}^{\alpha}} \frac{\partial u^{\tau}}{\partial \bar{u}^{\beta}}+\frac{\partial^{2} u^{\nu}}{\partial \bar{u}^{\alpha} \partial \bar{u}^{\beta}} .
\end{aligned}\right.
$$

Some fundamental tensor-invariants appear when we put the equations (1) and their equations of variation into an invariantive form by aid of the $D$ operator, keeping in mind that $p_{\alpha}^{d}$ is itself a fundamental invariant. For the differential operators, we note that $\partial / \partial p_{\alpha_{1}}^{i} \ldots \alpha_{q}$ is always a tensorial operator, and alternate this with $D_{\alpha}$, contracting for the index $\alpha$. This gives, on discarding any additive tensorial terms, a new operator in which the leading differentiation is of one order lower than in the previous case. The process may be repeated till we reach an operator which has only one (covariant) Latin index, all Greek indices having dropped out. This gives the complete set of differential operators associated with the equations (1); further alternations then give linear combinations of the same operators with coefficients which are the complete set of differential invariants of the space. The process amounts essentially to calculating the Poisson brackets for a system of linear partial differential equations of the first order, or the alternants of a Lie group, further theoretical justification being unnecessary in view of Hilbert's fundamental theorem on invariants.

In actual practice, the process has been carried out for $q=1$ and $q=2$, but is difficult even in spite of the simplified notation; generally mistakes occur which cannot be rectified. Therefore, some special choice of connection coefficients would be desirable. This is given by the following theorem.

THEOREM 1. The "best" intrinsic choice of connection (in the sense of reducing the amount of calculation) should be such that

$$
K_{\alpha \beta j}^{i} \equiv \partial_{\alpha} \gamma_{\beta j}^{i}-\partial_{\beta} \gamma_{\alpha j}^{i}+\gamma_{\beta j}^{\nu} \gamma_{\alpha \nu}^{i}-\gamma_{\alpha j}^{\nu} \gamma_{\beta \nu}^{i}=0
$$

The tensor $K_{\alpha \beta j}^{i}$ is therefore treated as an invariant of the connection here, merely for the sake of simplicity. But the vanishing of this invariant has more important consequences than just the reduction of bulk for our fundamental differential invariants. For this reason, I omit the actual calculations that prove Theorem 1 directly (particularly for the cases $q \geqq 2$ ) and take the further consequences as justification of the "best" choice.

The most important concept in classical differential geometry is 
the parallelism of Levi-Cività, defined originally for Riemannian spaces and immediately extended to paths with $m=1$. In that case, a vector $\lambda^{i}$ of the path-space is said to admit parallel displacement along a curve if $D \lambda^{i}=0$ along the curve. In our general case the "path" has to be regarded as a surface. Suppose we start with $n$ given components at an initial point and attempt, as in Riemannian geometry, to move the vector parallelly along a chosen path-base, here a "hypersurface," at least for $m<n$. We shall then have to solve

$$
\frac{\partial \lambda^{i}(u)}{\partial u^{\alpha}}+f_{\alpha \lambda}^{i} \lambda^{r}=0 ; \quad f_{\alpha j}^{i}=\gamma_{\alpha j}^{i} \text { along the base. }
$$

The conditions of integrability of (5) are, as usual,

$$
\lambda^{i}\left\{\frac{\partial f_{\beta j}^{i}}{\partial u^{\alpha}}-\frac{\partial f_{\alpha j}^{i}}{\partial u^{\beta}}+f_{\beta j}^{r} f_{\alpha r}^{i}-f_{\alpha i j}^{r} f_{\beta r}^{i}\right\}=0 .
$$

For proper unrestricted parallel displacement to be possible, the brackets must vanish for the given base. For a path-gometry, the least requirement is that displacement should be possible for any path as a base, seeing that the paths are necessary to define the operators $\partial_{\alpha}$, and hence $D_{\alpha}$, in (2). But, for a path, the braces in (6) are the tensor $K_{\alpha \beta j}^{i}$. This gives us the following theorem.

THEOREM 2. To admit unrestricted parallel displacement of a vector along a path-base, it is necessary and sufficient that $K_{\alpha \beta j}^{i}=0$ thereon.

This might have been regarded as an almost obvious restriction and the question then raised as to its importance, seeing that no such condition is needed for the $\Gamma_{\beta \nu}^{\alpha}$ coefficients of connection, that is, that parallelism along a path-base seems unnecessary for Greek-index vectors. This can be argued out at length by consideration of the difference between the dependent coordinates $x$ and the parameters $u$ that are our independent variables. But it seems more productive to prove:

THEOREM 3. It is possible to make the connection coefficients $\gamma_{\alpha\rfloor}^{4}=0$ along any chosen path-base if and only if $K_{\alpha \beta j}^{i}=0$ throughout.

This is the extension of the so-called theorem of Fermi to the pathspaces associated with partial differential equations. Its usefulness in shortening proofs and formal manipulations of differential geometry need not be explained. The actual proof is as follows. If it is possible to make $\gamma_{\alpha j}^{q}=0$ along any path, it then follows that $\partial_{\nu} \gamma_{\alpha j}^{s}=0$ also, as the operator is differentiation along the path and the connection coefficients vanish for some restricted but continuous region by hy- 
pothesis. Therefore, $K_{\alpha \beta j}^{i}=0$ for the chosen set of coordinates and the path in question. Now the vanishing of the tensor $K_{\alpha \beta j}^{i}$ does not depend upon the choice of coordinates. Furthermore, the path-space consists of the manifold swept out by the totality of paths, whence $K_{\alpha \beta \gamma}^{i}=0$ throughout the space. For the converse, if $K_{\alpha \beta \gamma}^{i}=0$, we can start with an ennuple of vectors $\lambda_{(j)}^{i}$ at a given point, and displace it along the path by parallel displacement. We know that by choosing $\operatorname{det} .\left|\lambda_{(j)}^{i}\right| \neq 0$ initially the ennuple will remain a set of $n$ independent vectors for some sufficiently restricted region. It only remains to set $\lambda_{(j)}^{j}=\partial x^{i} / \partial \bar{x}^{j}$ along the base of displacement, and this obviously admits infinitely many solutions for the transformations $\bar{x} \rightleftarrows x$, as the integrability condition is satisfied by hypothesis and we have imposed a set of initial conditions which depend upon the parameters $u$ only. It follows at once from the transformation laws (3) that $\bar{\gamma}_{\alpha j}^{q}=0$ on the base and this has been done by $x$-transformations alone.

It may be remarked in conclusion that $K_{\alpha \beta j}^{i}=0$ identically whenever there is a single independent variable, $m=1$. This covers Riemannian geometry, its extensions to symmetric affine connections, and the case of path-spaces for ordinary differential equations of any order.

REFERENCES. For the basic ideas and notation for ordinary pathspaces, D. D. Kosambi, Math. Zeit. vol. 37 (1933) pp. 608-618; Quart. J. Math., Oxford Ser. vol. 6 (1935) pp. 1-12; for partial differential equations J. Indian Math. Soc. vol. 3 (1939) pp. 249-253. It may be remarked that the work of $\mathrm{H}$. Hashimoto in calculating the invariants (J. Fac. Sci. Hokkaido Imp. Univ. vol. 8 (1940) pp. 163172 ) is actually in error. The final results will be published in a forthcoming paper by V. Seetharaman, Proceedings of the Indian Academy of Sciences.

Poona, India 